\title{
Desenvolvimento de um aplicativo mobile educacional para Medicina Nuclear
}

\author{
Development of an Educational Mobile App for Nuclear Medicine
}

\author{
Jaqueline De Santana Figueredo https://orcid.org/0000-0001-7820-1724 \\ Instituto Federal da Bahia - IFBA \\ E-mail: jaquellinemil@hotmail.com
}

\author{
Ítalo Luís Gomes Lacerda Dos Santos https://orcid.org/0000-0003-1198-7210 \\ Instituto Federal da Bahia - IFBA \\ E-mail: italolglds@gmail.com
}

Antônio Carlos Dos Santos Souza https://orcid.org/0000-0001-6593-3192

Instituto Federal da Bahia - IFBA

E-mail: antoniocarlos@ifba.edu.br

\author{
Wilson Otto Batista https://orcid.org/0000-0002-8464-5783 \\ Instituto Federal da Bahia - IFBA \\ E-mail: wilson.otto@ifba.edu.br
}

\section{Resumo}

Toda aprendizagem é mediada pelas tecnologias disponíveis em suas respectivas épocas, dessa forma, é necessário saber aliar os objetivos de ensino com os suportes tecnológicos que melhor atendam aos objetivos da aprendizagem. Na prática da Medicina Nuclear existem peculiaridades por utilizar radiação ionizante emitida de fontes não seladas, trazendo riscos de exposição e contaminação. Nota-se então a necessidade da aplicação de novas formas de tecnologia associadas à aprendizagem e ao ensino no campo de Medicina Nuclear. Nesse contexto foi criado um aplicativo educacional para dispositivos móveis, inicialmente compatível apenas para sistema Android, com o propósito de contribuir para a difusão do conhecimento sobre Medicina Nuclear e criação de uma interação em rede social de coautoria e confiança entre os interessados no tema, e podendo ampliar as possiblidades no contexto de ensino e aprendizagem da área. O sistema é dinâmico permitindo interação do usuário através de questionários, fóruns de discussões, perguntas e respostas, além de disponibilizar informações relevantes sobre Medicina Nuclear, de forma confiável e organizada, servindo para especialistas da área e também iniciantes. Dessa forma, o aplicativo visa contribuir para a área de ensino, visto que é de extrema importância a aplicação da ciência básica, dos requisitos de proteção radiológica e requisitos de boas práticas para alcançar uso seguro e prudente de materiais radioativos em seres humanos.

Palavras-chave: Medicina nuclear. Ensino. Disseminação de informação. Aplicativos móveis.

\begin{abstract}
Every learning is mediated by the technologies available in their respective times, therefore, it is necessary to know how to combine the teaching objectives with the technological supports that best live up the learning objectives. In the practice of Nuclear Medicine, both in its diagnostic and therapeutic aspects, the risks consist in exposure to the patient, to the worker and also the public, and contamination is fundamentally for the worker. In addition, there is still a risk to the environment through the radioactive
\end{abstract}


waste, if it's not properly managed. There is then a need to apply new forms of technology associated with learning and teaching in the field of Nuclear Medicine. In this context, the objective of this work was about the development of an educational application for mobile devices, with the purpose of contributing to the dissemination of knowledge about Nuclear Medicine and the creation of an interactive and trustful social network of co-authorship among stakeholders on the topic, being able to expand the possibilities in the context of teaching and learning in the field. The software is primarily aimed at students, teachers and professionals in Nuclear Medicine. However, due to the clear and concise language, the content is accessible to anyone who is interested. The system is dynamic, which allows the user an interaction through questionnaires, discussion forums, questions and answers, in addition to providing relevant information on Nuclear Medicine, in a reliable and organized way, serving both experts as well as for beginners. In this way, the application aims to contribute to the teaching area, since it is extremely important to apply basic science, radiological protection requirements and good practice requirements to achieve safe and prudent use of radioactive materials in humans.

Keywords: Nuclear medicine. Teaching. Dissemination of information. Mobile apps.

\section{Introdução}

Todo processo de aprendizagem pode ser mediado por tecnologias disponíveis em suas respectivas épocas, ou seja, é dinâmico e se modifica ao longo do tempo e de acordo com o desenvolvimento tecnológico do período. É preciso saber aliar os objetivos de ensino com os suportes tecnológicos que melhor atendam a esses objetivos (AGUIAR, 2008).

Educação e treinamento são processos pelo qual o saber, o conhecimento e as habilidades de uma geração são passados para a próxima. Hoje existem duas formas de educação e treinamento: educação convencional e educação à distância (MEHDIPOUR, 2013). O processo de ensino e aprendizagem são complexos, apresentam um caráter dinâmico e não acontece de forma linear como apenas um acúmulo de conhecimentos, deve ser uma tarefa de troca entre pessoas (MITRE, 2008).

$\mathrm{Na}$ área de saúde, o desenvolvimento tecnológico possibilitou novas perspectivas para a utilização das radiações em medicina, melhorando notavelmente a sua segurança e eficácia. Contudo, como em qualquer atividade humana, o uso incorreto ou inadequado de substâncias radioativas pode gerar riscos para a saúde (AZEVEDO, 2005).

A Medicina Nuclear é uma especialidade médica que utiliza radiação ionizante, pois faz uso de radioisótopos em sua rotina (ZEISSMANN; O'MALLEY; THRALL, 2014). $\mathrm{Na}$ prática da Medicina Nuclear, para diagnóstico ou terapia, os riscos são de exposição para o paciente, trabalhador e público, e de contaminação, fundamentalmente para o trabalhador. Além disso, ainda existe risco de contaminação para o meio ambiente, por meio dos rejeitos radioativos, se forem gerenciados de forma inadequada (MACHADO, 2010). Dessa forma, torna-se de extrema importância que os profissionais e /ou estudantes da Medicina Nuclear conheçam o funcionamento e consigam entender de forma clara todos os aspectos, riscos e particularidades do serviço para assim otimizar as práticas.

O cenário atual no processo de aprendizagem da área de Medicina Nuclear, se restringe basicamente aos materiais didáticos comuns, como livros e artigos científicos, deixando algumas lacunas em áreas especificas e para alguns profissionais da área. Diante do avanço das novas tecnologias e sua disponibilidade, 
vê-se então a necessidade de novas formas de aprendizagem e ensino ligadas à Medicina Nuclear, aumentando assim as possibilidades de métodos educacionais.

Nesse contexto, o objetivo deste trabalho foi o desenvolvimento de um aplicativo educacional para dispositivos móveis, com propósito de contribuir para a difusão do conhecimento sobre Medicina Nuclear, sob o ponto de vista dos conceitos básicos e proteção radiológica, e criação de uma interação em rede social de coautoria e confiança entre os interessados no tema. O software tem como público-alvo, preferencialmente, estudantes, docentes e profissionais de Medicina Nuclear, podendo alcançar outros interessados pela temática. O sistema é dinâmico permitindo interação do usuário através de questionários, fóruns de discussões, perguntas e respostas, além de disponibilizar informações relevantes sobre Medicina Nuclear, de forma confiável e organizada, servindo para especialistas da área e também iniciantes e podendo ampliar as possiblidades no contexto de ensino e aprendizagem da área.

\section{Metodologia}

O desenvolvimento do aplicativo foi estruturado conforme o diagrama apresentado na Figura 1. Iniciou-se com uma ampla revisão bibliográfica, abordando sobre Tecnologias associadas ao ensino e aprendizagem e sobre a Medicina Nuclear e busca por anterioridade junto as bases de dados INPI e google patentes. Posteriormente, foi decidido sobre a criação de um aplicativo mobile na área de Medicina Nuclear, garantindo a difusão do conhecimento.

Figura 1: Metodologia de Busca

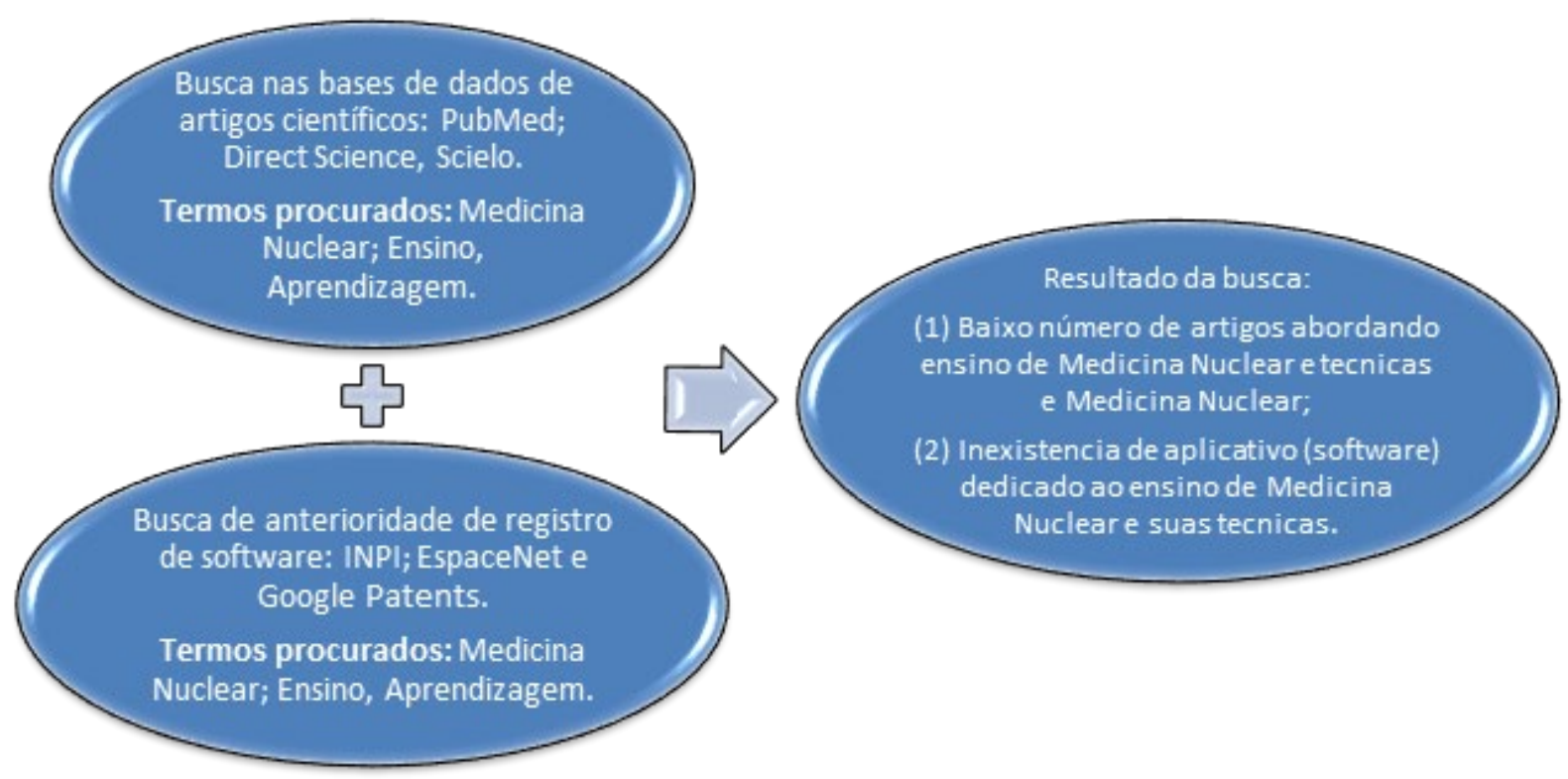

Fonte: Elaboração própria

Após análise dos resultados das buscas iniciou-se o desenvolvimento de um aplicativo compatível inicialmente, apenas para o sistema Android, disponível para download no Play Store. Podendo também ser acessado via web através do Blogger (apenas os conteúdos escritos como textos e artigos). O aplicativo possui basicamente as seguintes funcionalidades: 
(i). Postagens de assuntos sobre Medicina Nuclear

(ii). Questionários

(iii). Perguntas e Respostas/ Comentários

(iv). Discussão de casos (Fórum de Discussão)

(v). Convite para especialistas da área

O aplicativo foi construído na plataforma de desenvolvimento Android Studio. Em sua maioria, utilizando as linguagens de programação $J A V A^{T M}$ e XML. O Firebase utilizado para comunicação entre o aplicativo no dispositivo Android e o banco de dados. $O$ Android Studio e o Firebase são gratuitos.

Para a postagens dos conteúdos foram utilizadas também duas plataformas gratuitas, que são acessíveis e de fácil utilização. Para as postagens foi utilizado o Blogspot e para os questionários o Google Formulário. Ao serem postados nas seguintes plataformas já são diretamente direcionados para o aplicativo e ficam disponíveis para acesso do usuário cadastrado.

O aplicativo conta ainda com um sistema que contribui para uma rede de coautoria e confiança, pois permite que haja indicação entre os interessados no tema, através de convite, para contribuir com as informações no app.

Na revisão bibliográfica, detectamos a carência/inexistência de conteúdos práticos e de fóruns de discussão na área de Medicina Nuclear. Nessa área temos dois fatores destacáveis na questão de ensino e aprendizagem, pois trata de fatores intervenientes de um serviço de saúde e também com os referentes, a prática com uso de radiação ionizante.

Dentre os documentos analisados na pesquisa de anterioridade, um se destaca no ponto de vista da proteção radiológica e da formação continuada como ferramenta de garantia de qualidade de qualidade na transmissão de conhecimento para diferentes públicos. Este documento resume as recomendações da conferência de Bonn para proteção radiológica em medicina. Segundo a IAEA (2012, p. 2).

[...] não há dúvida de que a aplicação da radiação ionizante e de elementos radioativos em procedimentos de diagnóstico, intervenção ou terapêuticos em medicina é benéfico para centenas de milhões de pessoas todos os anos. No entanto, a utilização da radiação em medicina deve observar um equilíbrio entre os benefícios da melhoria do bem-estar e da saúde humana, bem como os riscos relacionados com a exposição dos indivíduos à radiação. Há a necessidade de uma abordagem holística que inclui parceria dos governos nacionais, da sociedade civil, organismos internacionais, investigadores, educadores, instituições e associações profissionais com o objetivo de identificar, defender e implementar soluções para enfrentar os desafios existentes e emergentes, além de liderarem, harmonizarem e coordenarem atividades e procedimentos a nível internacional

A conferência de Bonn publicou dez ações para a próxima década. Dentre elas destacamos as seguintes:

Ação 04: Fortalecer a educação e a formação dos profissionais de saúde em proteção radiológica.

Continuar a desenvolver a utilização das plataformas mais recentes, tais como as aplicações disponíveis na internet dedicadas ao treino, que cheguem a grupos profissionais mais alargados, para fins de formação.

Ação 08: Fortalecer a cultura de segurança radiológica na área da saúde. 
Melhorar o intercâmbio de informações entre pares sobre proteção radiológica e questões relacionadas com a segurança, utilizando os avanços das tecnologias de informação.

Ação 09: Fomentar um melhor diálogo sobre o risco-benefício no uso da radiação.

Aumentar a conscientização sobre os benefícios e os riscos da utilização da radiação entre profissionais de saúde, pacientes e público em geral (IAEA, 2012; p. 6:10-11).

Todas essas importantes ações estão contempladas na proposta do presente trabalho, conforme evidenciado nos tópicos disponibilizados no aplicativo educacional.

\section{Novas tecnologias e o processo de ensino e aprendizagem}

A aprendizagem não precisa ser apenas um processo de aquisição e domínio de conhecimentos. Pode se dar de forma coletiva e integrada, articulando informações e pessoas que estão em locais diferentes e que são de idade, sexo, condições físicas, áreas e níveis diferenciados de formação (KENSKI, 2003).

Como é fácil de verificar, aumentou de forma considerável a disponibilidade de software e de recursos educativos digitais (RAMOS, 2001). As atuais tecnologias digitais de comunicação e informação nos orientam para novas aprendizagens, que sejam caracterizadas como construções criativas, fluidas, mutáveis, que contribuindo para que as pessoas e a sociedade possam vivenciar pensamentos, comportamentos e ações criativas e inovadoras, que as encaminhem para novos avanços socialmente válidos no atual estágio de desenvolvimento da humanidade (KENSKI, 2003). Os ambientes e sistemas de ensino e aprendizagem integrados e abrangentes são capazes de promover o engajamento do usuário (AGUIAR, 2008).

A informação disponibilizada de forma digital é flexível, moldável, sujeita a alterações e permite a realização de várias atividades, visando o desenvolvimento de novas habilidades de aprendizagem, atitudes e valores pessoais e sociais. Criando assim, novos tempos e espaços educacionais, sendo novas formas de ensino em qualquer lugar, a qualquer hora são desenvolvidas a partir da necessidade de oferecer atualizações educacionais para todos. Em um tempo de mudanças rápidas, "o conhecimento científico-tecnológico desempenha um papel cada vez mais central como fator de mudanças e de dinamismo econômico e social" e exige que toda a sociedade se coloque em contínuo processo de aprendizagem (KENSKI, 2003).

A aprendizagem móvel é qualquer tipo de aprendizado que acontece quando o aluno não está em um local fixo e predeterminado, ou que aprende acontece quando o aluno aproveita as oportunidades de aprendizagem oferecidas pelas tecnologias móveis. Ou seja, com o uso de dispositivos móveis, os alunos podem aprender em qualquer lugar e a qualquer momento. Aprendizagem móvel é considerada a capacidade de usar dispositivos móveis para ensinar e aprender (MEHDIPOUR, 2013).

O aprendizado móvel está emergindo como uma das soluções para os desafios enfrentados pela educação. Com uma variedade de ferramentas e recursos sempre disponíveis, o aprendizado móvel fornece opções aumentadas para a personalização de aprendizagem (MEHDIPOUR, 2013). 


\section{Novas Tecnologias aplicadas ao ensino e aprendizagem dos profissionais da saúde}

Na formação dos profissionais de saúde, o processo educacional deve ter em vista o desenvolvimento tanto de capacidades gerais, identificadas com a grande área da saúde, quanto daquelas que constituem as especificidades de cada profissão (ALBUQUERQUE, 2008). Competências e habilidades precisam ser desenvolvidas pelos profissionais de saúde, durante e após a formação para que se constituam como sujeitos comprometidos com a busca de equidade do cuidado, do acesso e da cidadania (CHIESA, 2007).

A prática educativa em saúde refere-se tanto às atividades de educação em saúde, voltadas para o desenvolvimento de capacidades individuais e coletivas visando à melhoria da qualidade de vida e saúde; quanto às atividades de educação permanente, dirigidas aos trabalhadores da área de saúde através da formação profissional contínua (PEREIRA, 2003).

As tecnologias móveis são um meio atraente e fácil de manter habilidades de ensino e aprendizado, pois permite o acesso rápido às informações. São acessíveis, podem ser facilmente distribuídos e, portanto, têm um grande potencial para alcançar diversos usuários proporcionando-lhes acesso a mais aprendizagem e desenvolvimento. Tecnologias móveis podem facilitar a aprendizagem à distância em situações em que o acesso à educação é difícil ou interrompido pela localização geográfica, e podem ser acessadas no tempo preferível ou necessário (MEHDIPOUR, 2013).

Dessa forma, faz com que os profissionais de saúde tenham acesso tanto durante a sua formação, quanto durante o serviço ao surgir alguma dúvida sobre algum procedimento, tornando o acesso mais rápido e dinâmico, otimizando assim o serviço (CHIESA, 2007).

\section{Radiação ionizante na área médica}

As radiações que possuem energia suficiente para ionizar os átomos são chamadas de radiações ionizantes como é o caso dos raios $X$ e das radiações provenientes dos elementos radioativos, que são de origem nuclear, como as radiações alfa, beta e gama. As radiações ionizantes provêm de fontes naturais ou artificiais (NAVARRO, 2008). Durante toda a vida, os seres humanos estão expostos diariamente aos efeitos das radiações ionizantes. Estas radiações podem ser de origem natural ou artificial (AZEVEDO, 2005).

Em medicina, o uso das radiações ionizantes tem aplicações tanto no diagnóstico quanto na terapia. As grandes áreas de aplicação são: medicina nuclear, radioterapia e radiodiagnóstico (AZEVEDO, 2005).

A aplicação da radiação ionizante e de elementos radioativos em procedimentos de diagnóstico, intervenção ou terapêuticos em medicina é benéfica para diversas 
pessoas. No entanto, a utilização da radiação em medicina deve observar um equilíbrio entre os benefícios da melhoria do bem-estar e da saúde humana, bem como os riscos relacionados com a exposição dos indivíduos à radiação (SOARES; PEREIRA; FLÔR, 2011). Com isso a proteção radiológica busca um maior benefício efetivo aos usuários, profissionais e ambiente, sem limitação da prática (MACHADO, 2010).

A utilização da radiação ionizante na área médica vem expandindo e trazendo mais benefícios, assim como, possibilitando a detecção de tumores e fraturas (na radiografia convencional, tomografia computadorizada, mamografia), o tratamento de doenças (radioterapia e medicina nuclear), verificar a fisiologia dos órgãos e dos sistemas do corpo humano (medicina nuclear). Porém a interação da radiação com o tecido humano pode gerar efeitos biológicos, que foram notados logo após a descoberta da radiação $X$, quando surgiram doenças na pele das pessoas expostas aos raios $X$, levando cientistas a pesquisarem as possíveis causas (AZEVEDO, 2005).

Os efeitos causados pelas interações das radiações ionizantes com as células podem ocorrer de forma direta ou indireta. Na direta, uma macromolécula (DNA, proteínas, enzimas e outras) são danificadas, já na indireta, essa radiação interage com o meio e produz radicais livres. Essas modificações celulares podem ser reparadas através da ação de enzimas, porém se isso não ocorrer, surgirão lesões bioquímicas que podem causar danos (NAVARRO, 2008).

A manifestação dos efeitos biológicos ocorre de duas maneiras: o efeito determinístico, ocasionado por altas doses de radiação num curto espaço de tempo, e o efeito estocástico, provocado por pequenas doses recebidas em um longo período (SOARES; PEREIRA; FLÔR, 2011).

As primeiras recomendações de proteção para trabalhadores foram publicadas apenas 20 anos após a descoberta dos raios X, pela Rontgen Society, tendo em vista os inúmeros danos causados logo nos primeiros anos aos profissionais que a utilizavam. Esse foi o início do campo de estudo conhecido como proteção radiológica (NAVARRO, 2008).

A Proteção Radiológica tem como objetivo estabelecer normas adequadas de segurança e proteção para os seres humanos sem limitar indevidamente os benefícios de práticas que envolvam exposição a radiações (AZEVEDO, 2005).

\section{Medicina nuclear}

A Medicina Nuclear é definida pela OMS - Organização Mundial da Saúde como uma especialidade que se ocupa do diagnóstico, tratamento e investigação médica mediante ao uso de radioisótopos (BRASIL, 2015). Nela são utilizados os radiofármacos que são moléculas químicas ligadas a um elemento radioativo para assim serem utilizados como traçadores para diagnosticar disfunções ou patologias do organismo. Eles possuem a vantagem de não perturbarem a função quando administrados por via intravascular, diferentes de outras drogas diagnósticas. A 
radioatividade emitida pelo paciente é detectada por um equipamento chamado gama câmara, e a obtenção da imagem é dada através da conversão da radiação ionizante em energia elétrica, que é processada e gravada por computadores (ZEISSMANN; O'MALLEY; THRALL, 2014).

Existem fontes radioativas seladas e não seladas, as fontes seladas apresentam apenas o risco de exposição, uma vez que o material radioativo contido no recipiente não pode ser extraído. As práticas de proteção radiológica, nesse caso, incluem blindagens necessárias e serem armazenadas em local específico. Já as fontes não seladas ou abertas, que são as utilizadas em Medicina Nuclear além de riscos de exposição envolvem também risco de contaminação para a equipe, já que as fontes são manipuladas pelo trabalhador (MACHADO, 2010).

Por manipular fontes não seladas podem ocorrer derramamentos acidentais, que são classificados em categorias de menor e maior importância, dependendo da quantidade e do radionuclídeo, e irão necessitar de medidas de contenção diferentes. Sendo que todos os incidentes devem ser informados às instituições de controle e fiscalização (ZEISSMANN; O'MALLEY; THRALL, 2014). O contato continuo dos profissionais com a radiação ionizante, o tornam mais susceptíveis a uma maior exposição, o que traz a preocupação em relação a proteção já que acabam sendo expostos a radiação por longo tempo, mesmo que com pequenas doses, e essas podem ser nocivas à longo prazo (efeito estocástico) (SOARES; PEREIRA; FLÔR, 2011).

Por ser uma área onde o risco de exposição é alto, pelo uso das fontes não seladas, existe grande atenção à implantação de medidas de proteção radiológica que visem minimizar exposições desnecessárias aos pacientes, ao público, aos indivíduos ocupacionalmente expostos (IOE) e ao meio ambiente. Deve ser medida e documentada a quantidade e o tipo de radioatividade administrada ao paciente, e devem ser monitoradas as áreas do trabalho para manter a segurança dos pacientes e da equipe de trabalho (ZEISSMANN; O'MALLEY; THRALL, 2014).

Os IOE devem receber treinamento inicial em boas práticas de proteção radiológica em medicina nuclear e um treinamento periódico de proteção radiológica que deve ser oferecido pelo serviço (BRASIL, 2013), o que é fundamental para a redução de riscos e a prevenção de incidentes e acidentes com fortes não seladas (MENDES; FONSECA; CARVALHO, 2004).

\section{Novas tecnologias de ensino em medicina nuclear}

Dentre os pontos abordados na "Conferência Internacional de Proteção Contra Radiações em Medicina" realizado em Bonn - Alemanha, estão: Priorizar a educação e treino de todos profissionais de saúde em proteção radiológica, tendo em particular atenção os profissionais que utilizam radiação em medicina e em odontologia e aumentar a conscientização sobre os benefícios e os riscos da utilização da radiação 
entre profissionais de saúde, pacientes e público em geral (AZEVEDO, 2005).

O cenário atual no processo de aprendizagem da área de Medicina Nuclear, se restringe basicamente aos materiais didáticos comuns como livros e artigos científicos. Sendo que a maioria são voltados para a área de medicina ou de física médica, deixando assim, uma lacuna para com outros profissionais da área (HARAMITA, 2011).

Dessa maneira, vê-se a necessidade de abranger a Medicina Nuclear de forma objetiva e clara para todos os profissionais da área. Combinando várias ideias em um só produto, o que se caracteriza como inovação, como destacado por Baron e Shane (2010) ao dizer que "O que é novo é a combinação, não os componentes que fazem parte dela" e por Roger e Schoemaker (1971) afirmaram "que inovação é uma ideia, uma prática ou um objeto percebido como novo pelo indivíduo".

Portanto, na formação dos profissionais da área de Medicina Nuclear e mesmo na educação continuada dos profissionais surge a necessidade de utilização de mecanismos inovadores, que possibilitem o acesso fácil e o entendimento de forma dinâmica para que estejam tecnicamente preparados para saber lidar com a rotina do serviço. Para tanto, é necessário além de condições seguras de trabalho que haja treinamento e aptidões específicos para o exercício de suas funções.

\section{Aplicativo mobile educacional para medicina nuclear}

O aplicativo mobile educacional desenvolvido tem a seguinte configuração, como demostrado nas Figuras 2 a 3.

Figura 2: Tela Inicial do App

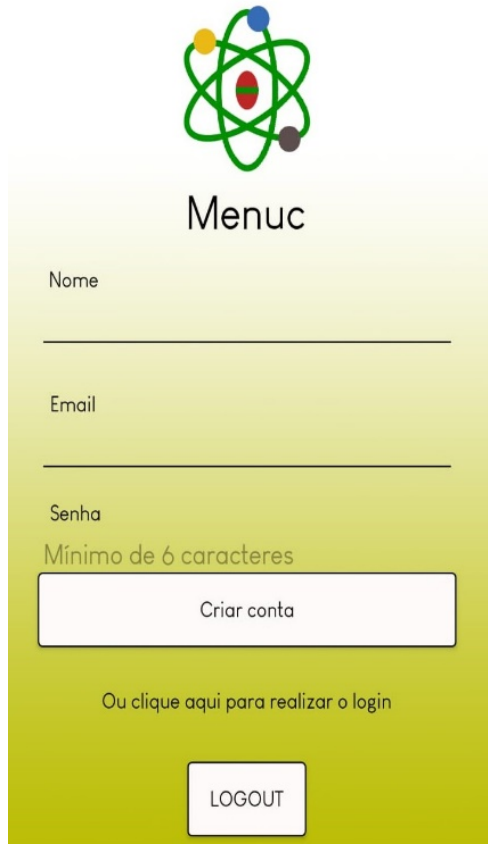

Fonte: Elaboração própria
Figura 3: Menu Principal do App

\section{MeNuc}

POSTAGENS

FÓRUM
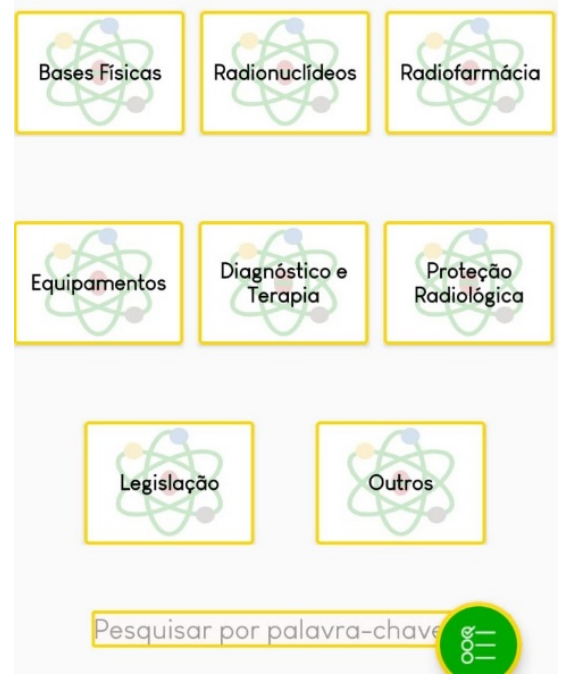

Fonte: Elaboração própria
Figura 4: Ampliação da Barra

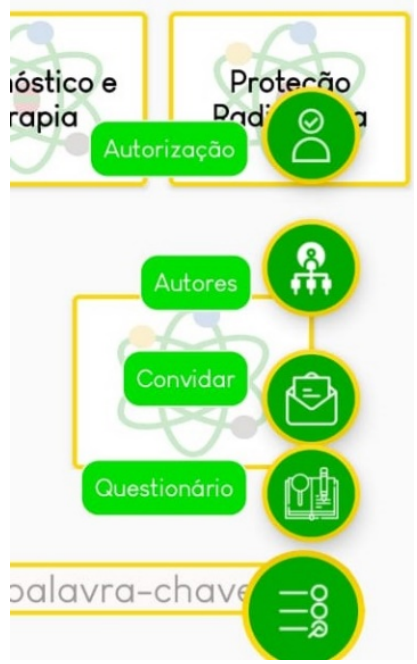

Fonte: Elaboração própria 
Essa ferramenta tem como objetivo geral contribuir para a difusão do conhecimento sobre Medicina Nuclear e criar uma interação em rede social entre os interessados no tema. E como objetivos específicos:

I. Disponibilizar informações relevantes sobre Medicina Nuclear, de forma confiável e organizada, servindo tanto para especialistas da área tanto para pessoas que estão iniciando interesse na área.

II. Garantir a integração entre os usuários acerca de Medicina Nuclear, utilizando rede social de coautoria e confiança.

III. Ser um aplicativo dinâmico, através de questionários, fóruns de discussões e perguntas e respostas referentes aos temas. Tendo como público-alvo estudantes, docentes, profissionais e demais interessados na área de Medicina Nuclear.

Entre as principais funcionalidades disponíveis no aplicativo, podemos citar:

\section{Postagens de assuntos sobre Medicina Nuclear}

As postagens realizadas no aplicativo são organizadas de acordo a subdivisão demonstrada abaixo, como forma de organização e facilidade para o usuário encontrar o que busca de forma mais direcionada. Todas contam com conceitos claros e objetivos, a fim de que possam ser compreendidos por públicos diferentes, com diferentes níveis de conhecimento prévio, já que terão usuários com diferentes necessidades. Além disso, os assuntos são divididos por nível de aprofundamento, visando sempre às boas práticas profissionais.

\section{Questionários}

O aplicativo conta com questionários elaborados com perguntas sobre os temas para testar conhecimento adquirido acerca dos temas abordados. Além disso também apresenta um questionário para solicitar autorização para contribuir com o aplicativo, ou seja, o usuário responde as questões e se atingir a nota mínima exigida, é autorizado a contribuir com o aplicativo.

\section{Perguntas e Respostas/ Comentários}

O usuário pode perguntar ou responder alguma dúvida ou questionamento referente aos temas abordados. Esse campo está disponível ao fim de cada postagem de assuntos. Os usuários autorizados podem responder os questionários

\section{Discussão de casos}

Com o intuito de promover interação entre os usuários, uma das ferramentas disponíveis refere-se ao fórum de discussão entre usuários, apresentando casos sobre Medicina Nuclear por usuários autorizados para então, promover uma discussão sobre o post.

\section{Convite para Especialistas da área}

A indicação de um outro usuário pode contribuir para uma rede social de coautoria e confiança, buscando um diferencial no aplicativo, permitindo interação de forma inovadora entre os usuários. 


\section{Considerações finais}

O processo de ensino e aprendizagem requer novos meios de inovação, de forma a acompanhar as novas tecnologias das épocas. O desenvolvimento tecnológico de forma acelerada vem emergindo em várias áreas, e modificando inclusive os processos na saúde e na educação.

$\mathrm{Na}$ área de saúde é de extrema importância que os profissionais conheçam as normas e o funcionamento com que estão lidando. A área de Medicina Nuclear, envolve o uso de radiação ionizante, tanto no diagnóstico quanto na terapia e utiliza fontes não seladas, trazendo assim, o risco de exposição e de contaminação, principalmente para os profissionais e meio ambiente. $\mathrm{O}$ uso apropriado dos fatores de proteção radiológica são ferramentas poderosas na redução da dose de exposição ocupacional.

Considerando que todo sistema está vulnerável a falhas, o que torna necessário treinamento e educação continuada dos profissionais, principalmente os que ficam em contato ao material radioativo, de forma a minimizar o risco de acidentes e aumento da exposição ocupacional, destaca-se a necessidade de novas possiblidades de contato com os conhecimentos e práticas educativas que visam contribuir com a área de ensino sobre esse tema, além de pacientes e o meio ambiente. Por isso é preciso que todos os profissionais envolvidos na rotina na área de saúde conheçam os riscos a que estão expostos e o funcionamento para saber como lidar caso ocorra algum acidente e/ ou imprevisto.

$\mathrm{Na}$ área de Medicina Nuclear temos dois fatores importantes na questão de ensino e aprendizagem dos envolvidos pois lida com os fatores por se tratar de um serviço de saúde e também com os fatores ao lidar com a radiação ionizante.

Vê-se então a necessidade de utilizar tecnologias existentes como apoio para o processo de ensino e aprendizagem acerca da área de Medicina Nuclear. Evidentemente que o futuro da aprendizagem móvel depende em grande parte do nível de aceitação social que recebe e do uso que os usuários fazem de tal tecnologia. É importante que a tecnologia seja utilizada sempre em associação com outros meios já disponíveis, e de forma consciente, em todas as áreas, inclusive na área de ensino e aprendizagem.

Dessa forma, acredita-se que o aplicativo pode contribuir para a difusão do conhecimento relacionado à temática, visto que é de extrema importância a aplicação da ciência básica, dos requisitos de proteção radiológica e requisitos de boas práticas para alcançar uso seguro e prudente de materiais radioativos em seres humanos. Estudos futuros irão avaliar a aplicação dessa ferramenta em diferentes contextos e assim então, será avaliada suas potencialidades, contribuições e limitações.

\section{Referências}

AGUIAR, E. V. B. As novas tecnologias e o ensino-aprendizagem. Vértices, v. 10, n. $1 / 3$, jan./dez. 2008.

ALBUQUERQUE, V. S., et al. A Integração Ensino-serviço no Contexto dos Processos de Mudança na Formação Superior dos Profissionais da Saúde. Revista Brasileira de Educação Médica. v. 32, n. 3 p. 356-362, Set. 2008.

AZEVEDO, A. C. P. Radioproteção em Serviços de Saúde. Programa de 
Radioproteção e Dosimetria da FIOCRUZ. SP, Instituto De Pesquisas Energéticas e Nucleares - IPEN, 2007.

BRASIL. Comissão Nacional de Energia Nuclear - Ministério Da Ciência, Tecnologia E Inovação. Disponível em: http://www.cnen.gov.br/. Acesso em: 18 set. 2021.

BRASIL. Norma Nuclear CNEN NN 3.05. Requisitos de segurança e proteção radiológica para serviços de medicina nuclear. Diário Oficial da Únião, Brasília, 2013.

CHIESA, A. M., et al. A Formação de Profissionais da Saúde: aprendizagem significativa à luz da promoção da Saúde. CogitareEnferm. 2007 Abr/Jun; v.12, n.2,p.236-40.

HARAMITA, T. Aplicação dos princípios de radioproteção em medicina nuclear Tamara Haramita. - Botucatu. Trabalho de conclusão de curso (bacharelado - Física Médica) - Instituto de Biociências de Botucatu, Universidade Estadual Paulista, 2011.

IAEA. 10 Actions to Improve Radiation Protection in Medicine in the Next Decade.[s.I.],2012.

Disponível em:https://www.iaea.org/sites/default/files/17/12/bonncall-for-action_por.pdf. Acesso em: 05 maio 2021.

KENSKI, V. M. Aprendizagem mediada pela tecnologia. Revista Diálogo Educacional, Curitiba, v. 4, n.10, p.47-56, set./dez. 2003.

MACHADO, M. A. D., et al. Revisão: Radioproteção aplicada à Medicina Nuclear. Revista Brasileira de Física Médica. v.4, n.3, p. 47-52, 2011. Disponível em: https://www.rbfm.org.br/rbfm/article/view/9. Acesso em: maio de 2021.

MEHDIPOUR, Y.; ZEREHKAFI, H. Mobile Learning for Education: Benefits and Challenges.International Journal of Computational Engineering Research, Vol, 03. Edição 6, 2013.

MITRE, S. M. Metodologias ativas de ensino-aprendizagem na formação profissional em saúde: debates atuais. Ciência \& Saúde Coletiva, v. 13(Sup 2):2133-2144, 2008.

MENDES, L. C. G; et al. Proposta de método de inspeção de radioproteção aplicada em instalações de medicina nuclear. Revista Radiologia Brasileira, vol. 37 (2): 11523, 2004.

NAVARRO, Marcus Vinicius Teixeira et al. Controle de riscos à saúde em radiodiagnóstico: uma perspectiva histórica. Hist. cienc. saude-Manguinhos, Rio de Janeiro , v. 15, n. 4, p. 1039-1047, 2008.

SOARES, F. A. P; PEREIRA, A. L e FLÔR, R. C. Utilização de vestimentas de proteção radiológica para redução de dose absorvida: uma revisão integrativa da literatura. Revista Radiologia Brasileira, vol. 44(2): 97-103, 2011.

PEREIRA, A. L. F. "As tendências pedagógicas e a prática educativa nas ciências da saúde." Cad. Saúde Pública, Rio de Janeiro, 19(5):1527-1534, set-out, 2003.

RAMOS, et al. Recursos educativos digitais: reflexões sobre a prática. Papert, 2001. THRALL, J. H.; ZIESSMAN, H. A. Medicina Nuclear. $2^{a}$ ed. Rio de Janeiro: Guanabara-Koogan, 2006. 
Recebido: $15 / 04 / 2021$

Aprovado: 03/11/2021

Como citar: FIGUEREDO, J. S. et al. Aplicativo Mobile Educacional para Medicina Nuclear.

Educitec - Revista de Estudos e Pesquisas sobre Ensino Tecnológico, v. 7, e171121, 2021.

Contribuição de autoria:

Jaqueline de Santana Figueredo: Conceituação, curadoria de dados, análise formal, investigação, metodologia, administração de projeto, escrita (rascunho original), escrita (revisão e edição).

Ítalo Luís Gomes Lacerda dos Santos: Curadoria de dados, investigação, software, validação.

Antônio Carlos dos Santos Souza: Curadoria de dados, investigação, supervisão, validação.

Wilson Otto Batista: Conceituação, curadoria de dados, investigação, metodologia, supervisão, escrita (revisão e edição), validação.

Direito autoral: Este artigo está licenciado sob os termos da Licença Creative CommonsAtribuição 4.0 Internacional 